\title{
Controlled Lateral Growth of Silica Nanowires and Coaxial Nanowire Heterostructures
}

\author{
R. G. Elliman,* T.-H. Kim, A. Shalav, and N. H. Fletcher \\ Department of Electronic Materials Engineering, Research School of Physics and Engineering, The Australian National University, \\ Canberra ACT 0200, Australia
}

ABSTRACT: Silica nanowires, grown on gold-coated silicon substrates by the active oxidation of silicon, are shown to undergo an initial stage of rapid longitudinal growth, followed by a stage of sustained lateral growth. During lateral growth, the average nanowire diameter increases linearly with annealing time and proceeds uniformly along the nanowire length at a rate of order $2 \mathrm{~nm} / \mathrm{min}$, thereby providing a simple and effective means of control. These observations are discussed with regard to predictions of a simple growth model based on the kinetic theory of ideal gases and are shown to provide a useful process for fabricating more complex silica-based coaxial core-shell heterostructures.

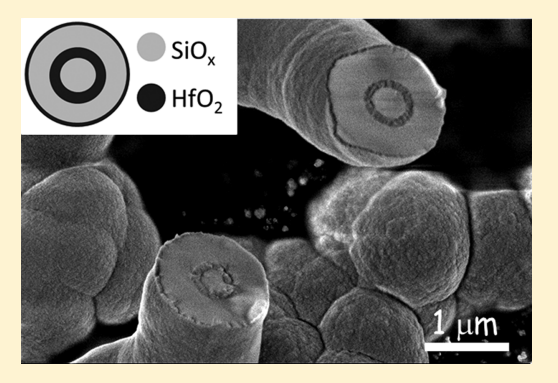

\section{INTRODUCTION}

Dense networks of substoichiometric silica $\left(\mathrm{SiO}_{x}\right)$ nanowires can be grown using a vapor-liquid-solid (VLS) mechanism in which a metal catalyst is used to absorb gaseous precursors containing silicon and oxygen. ${ }^{1-3}$ Interestingly, they can also be grown on metal-coated $\mathrm{Si}$ substrates without an external vapor source by annealing at higher temperatures under activeoxidation conditions. ${ }^{4,5}$ In this case, the growth mechanism is driven by the formation of volatile silicon monoxide gas, $\mathrm{SiO}(\mathrm{g})$, produced directly from the $\mathrm{Si}$ substrate by reactions with residual $\mathrm{O}_{2} \cdot{ }^{6-13}$ Nanowires fabricated by this method tend to have a convoluted surface morphology with a very large surface to volume fraction, properties that make them suitable as inert substrates for photocatalytic and biosensing applications. $^{14,15}$

Accurate control of nanowire dimensions is an important requirement for most practical applications as the physical and chemical properties of the nanowires can depend critically on their diameter, length, atomic composition, and density. ${ }^{16-18}$ For nanowires grown by a VLS process employing external vapor sources, this is generally achieved by selecting the size and distribution of catalyst particles, the type and concentration of the source gases, and the growth temperature or combinations of these parameters. ${ }^{16-19}$ This provides considerable flexibility and enables the growth of nanowires with different composition profiles and morphology. In contrast, the growth of nanowires from self-sourcing reactions, that is, those in which one or more of the source gases is produced by reaction with the substrate, provide much less control. ${ }^{8-12,15}$ Processes that offer additional means of control are therefore particularly useful in this case. In this study, we show that the self-sourcing active oxidation approach also provides a means of accurately controlling the average nanowire diameter. Our aim is to highlight the significant role of lateral growth in determining the nanowire diameter to show that lateral growth rate is a simple linear function of time, consistent with a simple model prediction, and to demonstrate how this can be exploited to embed functional layers within the nanowire structure.

\section{EXPERIMENTAL SECTION}

$\mathrm{SiO}_{x}$ nanowires were grown on commercially prepared (100) $\mathrm{Si}$ wafers (p-type, B-doped, $10-20 \Omega \cdot \mathrm{cm}$ ) by coating them with $0.1,1$, and $10 \mathrm{~nm}$ of Au by RF sputter-deposition and annealing them at $1100{ }^{\circ} \mathrm{C}$ in a quartz-tube (diameter: $4.5 \mathrm{~cm}$, length: $100 \mathrm{~cm}$ ) furnace purged with a high purity Ar gas with a flow rate of $2500 \mathrm{~mL} / \mathrm{min}$ at close to atmospheric pressure. Total anneal times ranged between $1 \mathrm{~min}$ and $1 \mathrm{~h}$ and were achieved by successive removal of individual samples after the specified time. The annealing gas contained $3-10 \mathrm{ppm}$ of $\mathrm{O}_{2}$ as a trace impurity (as specified by the distributor) and was dried prior to entering the furnace by passing it through a laboratory drying canister filled with anhydrous $\mathrm{CaSO}_{4}$. The furnace tube was sealed from the atmosphere by passing the purging gas through an oil-filled backflow preventer at the tube exit.

After growth, selected nanowire samples were subjected to postprocessing during which they were first conformally coated with a $50 \mathrm{~nm} \mathrm{HfO}_{2}$ layer using an atomic-layer deposition (ALD) technique, then reannealed for $1 \mathrm{~h}$ at $1100{ }^{\circ} \mathrm{C}$ under active oxidation conditions, and then recoated with a $25 \mathrm{~nm}$ $\mathrm{HfO}_{2}$ layer using ALD. ALD was undertaken using a Cambridge NanoTech Savannah System. Substrates were held at $200{ }^{\circ} \mathrm{C}$ during deposition, and the $\mathrm{HfO}_{2}$ layer was grown using alternating pulses of pure tetrakis-(dimethylamido)hafnium $\left(\mathrm{Hf}\left(\mathrm{NMe}_{2}\right)_{4}\right)$ and $\mathrm{H}_{2} \mathrm{O}$ vapor, with an $\mathrm{N}_{2}$ purge of the reaction chamber between pulses.

All samples were characterized by scanning electron microscopy (SEM) using either a Hitachi 4300 Schottky field-emission scanning electron microscope (FESEM) or a

\footnotetext{
Received: October 25, 2011

Revised: December 12, 2011

Published: January 6, 2012
} 
Zeiss UltraPlus analytical FESEM. Sample cross sections were prepared by mechanically cleaving the samples.

\section{RESULTS AND DISCUSSION}

The mechanisms responsible for silica nanowire growth have previously been described and are schematically summarized in Figure 1a-d. ${ }^{9,10}$ As a Au-coated silicon wafer is heated to 1100

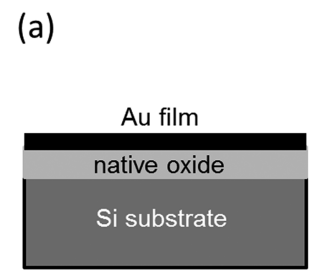

(c) Au tip evaporation

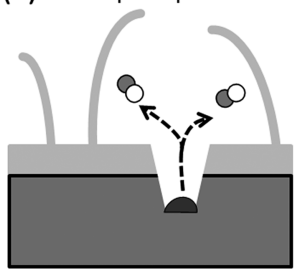

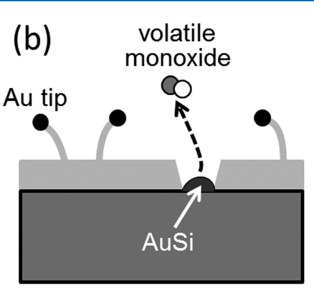

(d)

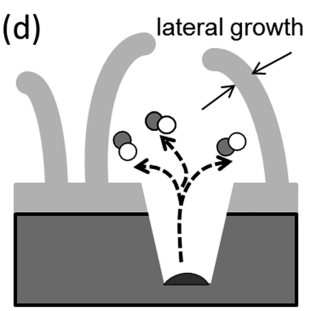

Figure 1. Temporal evolution of $\mathrm{SiO}_{x}$ nanowire growth via floatgrowth during active oxidation at $1100{ }^{\circ} \mathrm{C}$ showing the (a) as prepared sample, (b) nanowire nucleation and growth, (c) catalyst evaporation, and (d) lateral growth at prolonged annealing times.

${ }^{\circ} \mathrm{C}$, catalyst particles form on the wafer surface by islanding of the deposited film. These Au particles act as catalysts for the decomposition of the native oxide ${ }^{20}$ and once in contact with the $\mathrm{Si}$ enhance the active oxidation process, ${ }^{21}$ creating etch pits and a volatile monoxide vapor, $\mathrm{SiO}(\mathrm{g})$, via the reaction ${ }^{22}$

$$
2 \mathrm{Si}(\mathrm{s})+\mathrm{O}_{2}(\mathrm{~g}) \rightarrow 2 \mathrm{SiO}(\mathrm{g})
$$

where (s) and (g) denote the solid and gaseous phases, respectively. Au-rich nanoparticles remaining on the native oxide, or Au-rich particles formed within the etch pits, act as catalysts for $\mathrm{SiO}_{x}$ nanowire growth via the VLS mechanism with $\mathrm{SiO}(\mathrm{g})$ as the precursor gas. Once nucleated, $\mathrm{SiO}_{x}$ nanowires undergo three distinct stages of growth: (1) rapid longitudinal growth (Figure 1b), (2) slower longitudinal growth during which the Au catalyst is depleted (Figure 1c), and (3) sustained lateral growth as the nanowires continue to be bathed in $\mathrm{SiO}$ vapor (Figure 1d). The effect of this latter stage of growth is evident in Figure 2, which shows SEM micrographs of sample cross sections $(10 \mathrm{~nm} \mathrm{Au}$ on $\mathrm{Si})$ after annealing at a
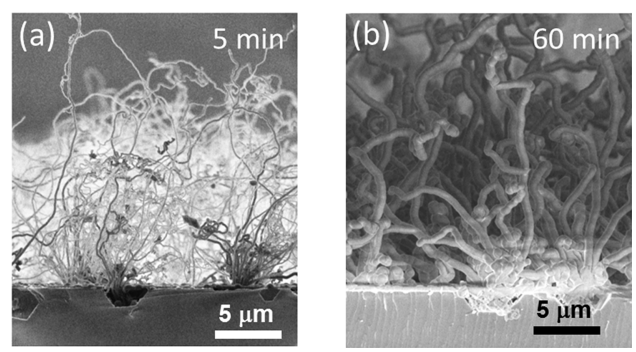

Figure 2. Typical cross-section SEM micrographs after (a) a 5 min anneal and (b) a 60 min anneal for samples coated with a $10 \mathrm{~nm} \mathrm{Au}$ film. These images correspond with the schematics depicted in Figure $1 \mathrm{c}, \mathrm{d}$, respectively. temperature of $1100{ }^{\circ} \mathrm{C}$ for 5 (Figure 2a) and $60 \mathrm{~min}$ (Figure $2 b)$. Comparison of the two Figures clearly shows that there is an increase in nanowire diameter with increasing annealing time. In general, however, silica nanowire growth also depends on the thickness of the deposited $\mathrm{Au}$ film because this determines the size and density of the catalyst particles, which in turn affects their reactivity and catalytic activity.

Figure 3 shows average nanowire diameters as a function of annealing time for three different Au layer thicknesses. This shows that the average nanowire diameter increases approx-

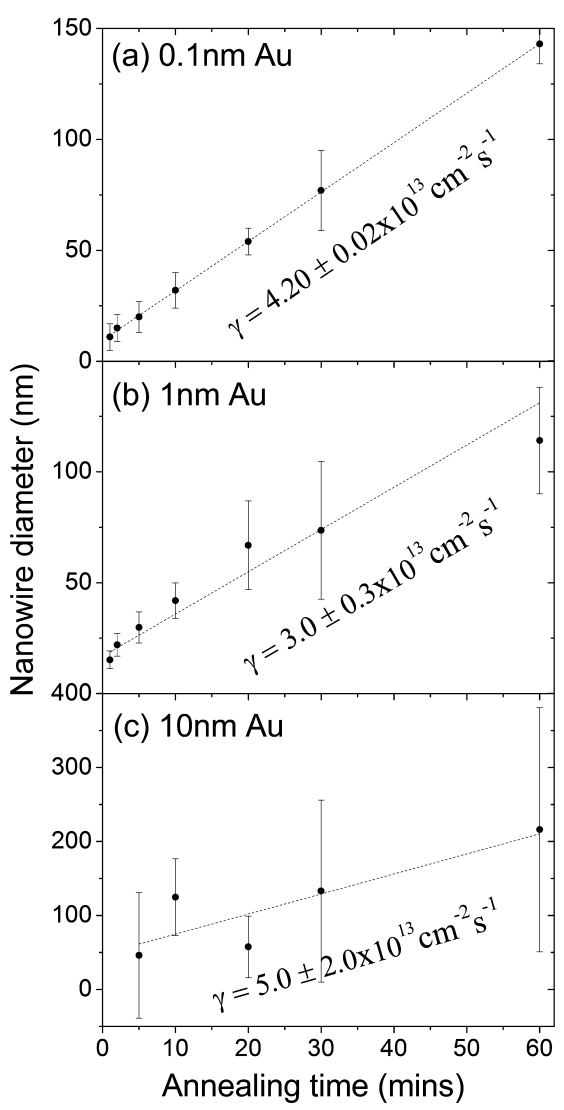

Figure 3. $\mathrm{SiO}_{x}$ nanowire diameter as a function of annealing time for samples coated with Au layers of thickness: (a) 0.1 , (b) 1 , and (c) 10 $\mathrm{nm}$. The error bars represent the full width at half-maximum (fwhm) values for Gaussian fits to the diameter distributions after a given annealing time, and $\gamma$ is the molecular flux calculated from the model described in the text.

imately linearly with annealing time for Au-layer thicknesses ranging from 0.1 to $10 \mathrm{~nm}$. For the thinnest gold layer, where the average Au particle size is smallest, the nanowire diameter is reasonably uniform, and extrapolation of the wire diameter to zero annealing time yields a diameter of $9.5 \pm 0.4 \mathrm{~nm}$, close to the theoretical minimum diameter for silica nanowire growth of $\sim 10 \mathrm{~nm}^{8}$. However, for thicker layers, there is an increasing spread in nanowire diameters, including the development of bimodal size distributions that appear to result from a second generation of nanowire nucleation.

Because the dominant gas-phase reactants in the furnace are $\mathrm{SiO}(\mathrm{g})$ and $\mathrm{O}_{2}(\mathrm{~g})$, lateral growth is assumed to proceed via the reaction

$$
2 \mathrm{SiO}+\mathrm{O}_{2}(\mathrm{~g}) \rightarrow 2 \mathrm{SiO}_{2}(\mathrm{~s})
$$


This reaction can be interpreted in two distinct ways, either as

$$
\mathrm{SiO}+\mathrm{SiO} \rightarrow \mathrm{SiO}_{2}+\mathrm{Si}
$$

followed by

$$
\mathrm{Si}+\mathrm{O}_{2} \rightarrow \mathrm{SiO}_{2}
$$

or, alternatively, as

$$
\mathrm{SiO}+\mathrm{O}_{2} \rightarrow \mathrm{SiO}_{2}+\mathrm{O} \uparrow
$$

with atomic oxygen potentially reentering the reaction chain through various reactions, including $\mathrm{SiO}+\mathrm{O} \rightarrow \mathrm{SiO}_{2}$, or $\mathrm{O}+\mathrm{O}$ $\rightarrow \mathrm{O}_{2}$.

This involves the production of $\mathrm{SiO}(\mathrm{g})$ at the wafer surface, its diffusive transport up through the forest of nanowires, and its reaction on the nanowire surface to produce $\mathrm{SiO}_{x}$, with $x$ having a typical value of $\sim 1.8^{6}$. Detailed and precise modeling of this growth process can clearly only be undertaken numerically, and the results would apply only to the specific arrangement calculated, so that this does not add greatly to the understanding of the processes involved. For this reason, it is appropriate to examine somewhat simplified models. The following discussion outlines a simple model based on lowprobability gas adsorption on cylindrical nanowires. A more detailed model based on diffusive transport of gas-phase reactants is presented in Appendix A.

Assuming that the nanowires are bathed in a vapor source of reactants that produces lateral growth via molecular adsorption reactions on the nanowire surface and that the rate of adsorption is small compared with the concentration of reactants, then the reactant concentration at the nanowire surface can be taken to be that in the ambient. For a cylindrical nanowire with radius $r$, volume $V$, and length $l$, simple algebra then shows that $\mathrm{d} V / \mathrm{d} t=\gamma A / \rho$, where $\gamma$ is the molecular flux adsorbing on the cylinder surface of area $A$, and $\rho$ is the molecular density of the resulting $\mathrm{SiO}_{x}$ layer. Given that the volume of the cylinder is $V=\pi r^{2} l$ and the surface area is approximately $A=2 \pi r l$ (ignoring the ends of the cylinder), then the cylinder radius is approximately $r=\gamma t / \rho$ and increases linearly with time, which is what is observed experimentally. Fitting this dependence to the data of Figure 2 and assuming a molecular density for $\mathrm{SiO}_{2}$ of $2.2 \times 10^{22} \mathrm{~cm}^{-3}$, gives a value of $\gamma$ $=(4.20 \pm 0.02),(3.0 \pm 0.3)$, and $(5.0 \pm 2.0) \times 10^{13} \mathrm{~cm}^{-2} \cdot \mathrm{s}^{-1}$ for the molecular adsorption rate for the $0.1,1$, and $10 \mathrm{~nm} \mathrm{Au}$ coated samples, respectively.

It is instructive to compare these numbers with estimates from the kinetic theory of ideal gases, which predicts that the flux of molecules hitting a surface at atmospheric pressure and a temperature of $1100{ }^{\circ} \mathrm{C}$ is on the order of $10^{23} \mathrm{~cm}^{-2} \cdot \mathrm{s}^{-1}$. From the values $\gamma$ determined above this suggests that about 1 molecule in $10^{10}$ that hits the nanowires surface contributes to lateral growth. For an ideal bimolecular reaction and unit sticking coefficient this would imply a gas-phase reactant concentration on the order $10^{-5}$ (i.e., $10 \mathrm{ppm}$ ), which is on the order of the $\left[\mathrm{O}_{2}\right]$ concentration in the annealing ambient. However, because reactions $3 \mathrm{a}, 3 \mathrm{~b}$, and 4 are mediated by adsorption of $\mathrm{SiO}(\mathrm{g})$ on the nanowire surface, the exact reaction kinetics are more likely described by the LangmuirHinshelwood and Eley-Rideal mechanisms, respectively. ${ }^{23}$

Although detailed modeling of the reaction kinetics is beyond the scope of the present study, it is worth noting that the two reactants, $\mathrm{SiO}(\mathrm{g})$ and $\mathrm{O}_{2}(\mathrm{~g})$, diffuse through the nanowire network in opposite directions, and as a consequence the product of their concentrations is approximately constant within the nanowire layer. The reaction rate within the nanowire layer is therefore expected to be more uniform than the individual reactant concentrations and to result in nearuniform lateral growth along the nanowire length. (For example, if reaction 4 was to dominate, then with $\mathrm{O}$ lost to the ambient, the reaction rate would scale as $[\mathrm{SiO}]\left[\mathrm{O}_{2}\right]$ and would be approximately uniform throughout the nanowire layer). Figure 4a shows an SEM cross-section of a sample (10

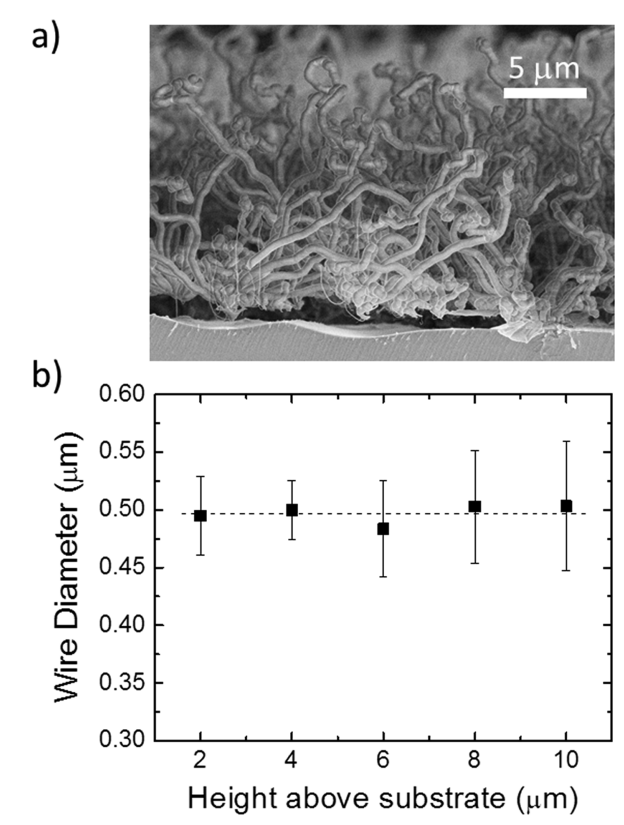

Figure 4. (a) SEM cross-section of a sample coated with $10 \mathrm{~nm}$ of $\mathrm{Au}$ and annealed for $1 \mathrm{~h}$ at $1100{ }^{\circ} \mathrm{C}$. (b) Average nanowire diameter as a function of height above the substrate, determined from panel a.

$\mathrm{nm} \mathrm{Au}$ film on $\mathrm{Si}$ ) annealed for $60 \mathrm{~min}$, showing that the average diameter of the nanowires is close to $500 \mathrm{~nm}$, consistent with the wires having undergone extensive lateral growth. Measurements of the average nanowire diameter as a function of height above the Si surface are shown in Figure $4 \mathrm{~b}$ and show that the diameter is invariant over distances of $10 \mu \mathrm{m}$ above the Si surface, consistent with these expectations.

The use of lateral growth to control the diameter of nanowires is clearly very useful but also raises the possibility of fabricating more complex multishell heterostructures. As an example, Figure 5 shows a $\mathrm{SiO}_{x} / \mathrm{HfO}_{2} / \mathrm{SiO}_{x} / \mathrm{HfO}_{2}$ multishell heterostructure fabricated by successive active oxidation and ALD deposition. The initial $\mathrm{SiO}_{x} \mathrm{NWs}$ were grown as described above (i.e., by depositing a $10 \mathrm{~nm}$ Au layer on (100) Si and annealing for $1 \mathrm{~h}$ at $1100{ }^{\circ} \mathrm{C}$ in an ambient containing 3-10 ppm $\left.\mathrm{O}_{2}\right)$. These were then coated with a thin $(50 \mathrm{~nm})$ conformal layer of $\mathrm{HfO}_{2}$ using atomic layer deposition (ALD) and reannealed under active oxidation conditions ( $1 \mathrm{~h}$ at 1100 $\left.{ }^{\circ} \mathrm{C}\right)$ to deposit a $\mathrm{SiO}_{x}$ shell-layer. A final conformal layer (25 $\mathrm{nm})$ of $\mathrm{HfO}_{2}$ was then deposited by ALD to delineate clearly the deposited $\mathrm{SiO}_{x}$ layer.

\section{SUMMARY AND CONCLUSIONS}

$\mathrm{SiO}_{x}$ nanowires grown under active oxidation conditions were shown to undergo a stage of sustained lateral growth that resulted in a near-uniform increase in nanowire diameter along their length. The increase in nanowire diameter was shown to be linear with annealing time, consistent with the predictions of 

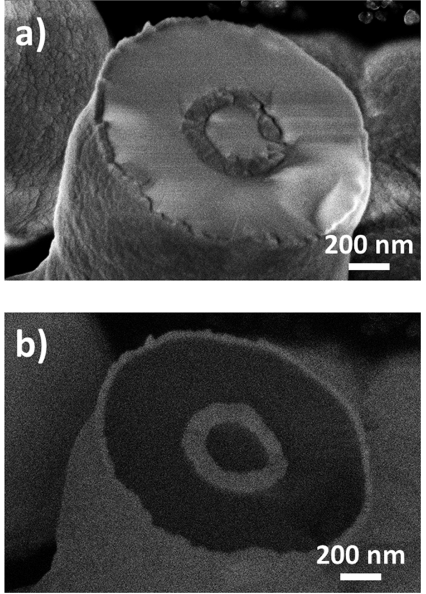

Figure 5. SEM images of a fractured nanowire consisting of a coaxial $\mathrm{SiO}_{x}$ core, a $50 \mathrm{~nm} \mathrm{HfO}_{2}$ cladding layer, an $\mathrm{SiO}_{x}$ cladding layer deposited by lateral growth, and a $25 \mathrm{~nm}$ outer $\mathrm{HfO}_{2}$ cladding layer. (a) Secondary electron image and (b) electron backscatter image.

a simple growth model based on gas-phase transport of reactants and to proceed at a rate of order $2 \mathrm{~nm} / \mathrm{min}$. This observation enables a simple estimate of the nanowire diameter for different annealing times and demonstrates that a basic absorption process can account for the observed behavior; that is, effects such as surface diffusion do not appear to play a significant role. Finally, it was shown that the lateral growth of $\mathrm{SiO}_{x}$ can be exploited to fabricate more complex coaxial heterostructures, demonstrating that additional functional layers can be readily incorporated on or embedded within the nanowire structure.

\section{APPENDIX A}

If the reaction rate on the nanowire surface is sufficient to deplete the surrounding $\mathrm{SiO}$ concentration, then a more sophisticated lateral-growth model is required. Assuming that nanowires tend to grow so that they maintain approximately equal spacing from their neighbors, each wire can be thought of as growing along the axis of a bent polyhedral tube, the surface of which is defined as equidistant from the wire in question and its nearest neighbors. Such a structure is analogous to the Voronoi polyhedra commonly used in examining complex materials. ${ }^{24}$ The reaction product of the $\mathrm{SiO}(\mathrm{g})$ and $\mathrm{O}_{2}(\mathrm{~g})$ concentrations may then be assumed to be maintained at a particular value on the surface of the irregular columnar polyhedron surrounding each wire.

Because a given nanowire runs along the axis of a bent polygonal tube of approximately constant radius, the obvious simplifications are to replace the bent tube by a straight one and to approximate its polygonal boundary by a cylinder of radius $R$ with the wire along its axis. The chemical force driving wire growth, which is essentially the product of the concentrations of $\mathrm{O}_{2}(\mathrm{~g})$ and $\mathrm{SiO}(\mathrm{g})$, can then be taken to be constant on the surface of this cylinder. Growth behavior in this simple situation is now relatively easy to calculate.

In a quasi-steady-state situation, the $\mathrm{SiO}(\mathrm{g})$ and $\mathrm{O}_{2}(\mathrm{~g})$ vapor pressure $p(r)$ in the space surrounding the wire must satisfy the equation $\nabla^{2} p=0$ or because of the cylindrical symmetry and assumed lack of variation along the wire the equation

$$
\frac{1}{r} \frac{\mathrm{d}}{\mathrm{d} r}\left(r \frac{\mathrm{d} p}{\mathrm{~d} r}\right)=0
$$

The solution is $\mathrm{d} p / \mathrm{d} r=A / r$, which can be integrated to give

$$
p(r)=A \log (r / R)+B
$$

with $A$ and $B$ being constants. Using the boundary conditions that $p(R)=p_{1}$ and $p(a)=p_{0}$, where $a$ is the radius of the wire and $p_{0}$ is the saturation vapor pressure of $\mathrm{SiO}(\mathrm{g})$ and $\mathrm{O}_{2}(\mathrm{~g})$ at the wire surface, then leads to the result

$$
p(r)=p_{1}-\left(p_{1}-p_{0}\right) \frac{\log (r / R)}{\log (a / R)}
$$

The rate of radial growth of the wire is proportional to the pressure gradient $\mathrm{d} p / \mathrm{d} r$ at its surface so that

$$
\frac{\mathrm{d} a}{\mathrm{~d} t}=-\frac{A\left(p_{1}-p_{0}\right)}{a \log (a / R)}
$$

where $A$ is proportional to the diffusion constant in the vapor and inversely proportional to the molecular density in the solid.

This equation can be written in the simpler form

$$
\frac{\mathrm{d} x}{\mathrm{~d} \tau}=\frac{-1}{x \log x}
$$

with $x=a / R$ and $\tau=A t / R^{2}$, and this equation can actually be solved explicitly ${ }^{25}$ as

$$
x(\tau)=2\left[\frac{-\tau}{W(-4 \tau / e)}\right]^{1 / 2}
$$

where $W(x)$ is the little-known Lambert $W$ function $^{26,27}$ introduced by J. H. Lambert ${ }^{28}$ in 1758 .

A plot of this equation, Figure A1, shows that for very thin wires $(x<0.05)$ the radius increases as about $\tau^{1 / 2}$, reflecting the

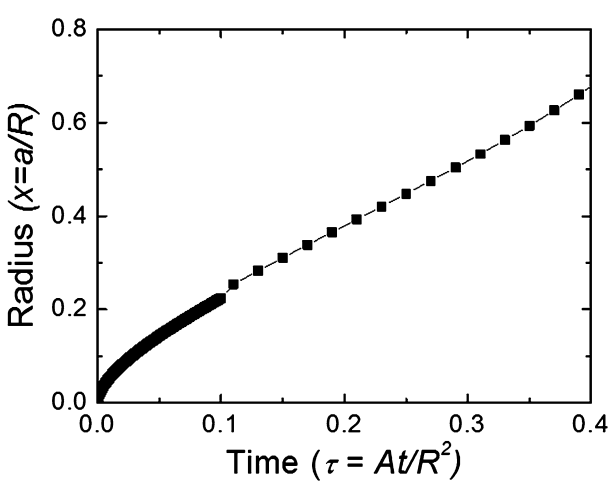

Figure A1. Plot of eq A6.

fact that growth of the wire has very little effect on the overall diffusion field. For thicker wires $(0.1<x<0.7)$, the variations of $x$ and $\log x$ in the denominator of A5 nearly cancel, giving near-linear growth with $\mathrm{d} x / \mathrm{d} \tau$ approximately constant. (Note that there is an unrealistic increase in growth rate for larger diameter wires (as $x \rightarrow 1$ ) because the model assumes that the concentration of $\mathrm{SiO}(\mathrm{g})$ and $\mathrm{O}_{2}(\mathrm{~g})$ is maintained within the very narrow spaces between growing wires, which is unphysical.) Predictions from the theory should therefore be limited to the region where $a$ is less than about $0.7 R$. 
Rather than using this complicated function, however, it is simpler to integrate eq A5 numerically, from which it is clear that there is an approximately linear relation

$$
x(\tau) \approx 0.1+1.4 \tau
$$

for $0.1<\tau<0.4$. For comparison with experiment, it is best to translate this relation between $x$ and $\tau$ back to the physical variables $a$, $t$, and $R$. The linear approximate relation for A7 then becomes

$$
a(t) \approx 0.1 R+\frac{1.4 A}{R} t
$$

This predicts the linear time dependence observed experimentally and additionally shows that the rate of growth $\mathrm{d} a / \mathrm{d} t$ is inversely proportional to the interwire spacing $2 R$ but is nearly independent of the wire radius $a$ over the range $0.1 R<a$ $<0.7 R$.

\section{AUTHOR INFORMATION}

\section{Corresponding Author}

*E-mail: rob.elliman@anu.edu.au. Tel: +61 261250521.

\section{REFERENCES}

(1) Fang, X. S.; Zhang, L. D. J. Mater. Sci. Tech. 2006, 22, 1-18.

(2) Cheong, K. Y.; Chiew, Y. L. Advances of $\mathrm{SiOx}$ and $\mathrm{Si} / \mathrm{SiOx}$ coreshell nanowires. In Nanowires Science and Technology; Lupu, N., Ed.; INTECH, 2010; Vol. 12, pp 131-150.

(3) Sekhar, P. K.; Bhansali, S. Mater. Lett. 2010, 64, 729-732.

(4) Liu, Z. Q.; Xie, S. S.; Sun, L. F.; Tang, D. S.; Zhou, W. Y.; Wang, C. Y.; Liu, W.; Li, Y. B.; Zou, X. P.; Wang, G. J. Mater. Res. 2001, 16, 683-686.

(5) Paulose, M.; Varghese, O. K.; Grimes, C. A. J. Nanosci. Nanotechnol. 2003, 3, 341-346.

(6) Sekhar, P. K.; Samhandam, S. N.; Sood, D. K.; Bhansali, S. Nanotechnology 2006, 17, 4606-4613.

(7) Pan, Z. W.; Dai, Z. R.; Ma, C.; Wang, Z. L. J. Am. Chem. Soc. 2002, 124, 1817-1822.

(8) Hourlier-Bahloul, D.; Perrot, P. C. R. Chim. 2007, 10, 658-665.

(9) Shalav, A.; Kim, T.; Elliman, R. G. IEEE J. Sel. Top. Quantum Electron. 2011, 17, 785-793.

(10) Kim, T. H.; Shalav, A.; Elliman, R. G. J. Appl. Phys. 2010, 108, 076102.

(11) Sekhar, P. K.; Wilkinson, A. R.; Elliman, R. G.; Kim, T. H.; Bhansali, S. J. Phys. Chem. C 2008, 112, 20109-20113.

(12) Elliman, R. G.; Wilkinson, A. R.; Kim, T. H.; Sekhar, P. K.; Bhansali, S. J. Appl. Phys. 2008, 103, 104304-1-104304-5.

(13) Shalav, A.; Collin, G. H.; Yang, Y.; Kim, T.; Elliman, R. G. J. Mater. Res. 2011, 26, 2240-2246.

(14) LaLonde, A. D.; Norton, M. G.; Zhang, D. Q.; Gangadean, D.; Alkhateeb, A.; Padmanabhan, R.; McIlroy, D. N. J. Mater. Res. 2005, 20, 3021-3027.

(15) Sekhar, P. K.; Ramgir, N. S.; Bhansali, S. J. Phys. Chem. C 2008, $112,1729-1734$.

(16) Wang, N.; Cai, Y.; Zhang, R. Q. Mater. Sci. Eng., R 2008, 60, 151.

(17) Kuchibhatla, S.; Karakoti, A. S.; Bera, D.; Seal, S. Prog. Mater. Sci. 2007, 52, 699-913.

(18) Lu, W.; Lieber, C. M. J. Phys. D: Appl. Phys. 2006, 39, R387R406.

(19) Barth, S.; Hernandez-Ramirez, F.; Holmes, J. D.; RomanoRodriguez, A . Prog. Mater. Sci. 2010, 55, 563-627.

(20) Dallaporta, H.; Liehr, M.; Lewis, J. E. Phys. Rev. B 1990, 41, 5075-5083.

(21) Cros, A.; Salvan, F.; Commandre, M.; Derrien, J. Surf. Sci. 1981, 103, L109-L114.

(22) Gelain, C.; Cassuto, A.; Legoff, P. Oxid. Met. 1971, 3, 139-\&.
(23) Chorkendorff, I.; Niemantsverdriet, J. W. Concepts of Modern Catalysis and Kinetics; Wiley-VCH: Weinheim, Germany, 2003; p 452. (24) Brostow, W.; Chybicki, M.; Laskowski, R.; Rybicki, J. Phys. Rev. B 1998, 57, 13448-13458.

(25) WolframAlpha website. www.wolframalpha.comAug 25, 2009.

(26) Corless, R. M.; Gonnet, G. H.; Hare, D. E. G.; Jeffrey, D. J.; Knuth, D. E. Adv. Comput. Math. 1996, 5, 329-359.

(27) Valluri, S. R.; Jeffrey, D. J.; Corless, R. M. Can. J. Phys. 2000, 78, 823-831.

(28) Lambert, J. H. Acta Helvitica physico-mathematico-anatomicobotanico-medica 1758, 3, 128-168. 